\title{
Acknowledgment to Reviewers of Applied System Innovation in 2020
}

\author{
Applied System Innovation Editorial Office
}

Citation: Applied System Innovation Editorial Office. Acknowledgment to Reviewers of Applied System Innovation in 2020. Appl. Syst. Innov. 2021, 4, 7. https://doi.org/10.3390/ asi4010007

Published: 26 January 2021

Publisher's Note: MDPI stays neutral with regard to jurisdictional claims in published maps and institutional affiliations.

Copyright: () 2021 by the author. Licensee MDPI, Basel, Switzerland. This article is an open access article distributed under the terms and conditions of the Creative Commons Attribution (CC BY) license (http://creativecommons.org/licenses /by/4.0/).
MDPI AG, St. Alban-Anlage 66, 4052 Basel, Switzerland

Peer review is the driving force of journal development, and reviewers are gatekeepers who ensure that Applied System Innovation maintains its standards for the high quality of its published papers. Thanks to the cooperation of our reviewers, in 2020, the median time to first decision was 15 days and the median time to publication was 42 days. The editors would like to express their sincere gratitude to the following reviewers for their precious time and dedication, regardless of whether the papers were finally published:

\begin{tabular}{|c|c|}
\hline Abbas, Azhar & Črešnar, Rok \\
\hline Abdul, Majeed & Cristescu, Marian Pompiliu \\
\hline Abreu, António & Cuculić, Aleksandar \\
\hline Aguasca-Colomo, Ricardo & Czerwinski, Robert \\
\hline Ahmed, Mohiuddin & Datta, Arka \\
\hline Alessandro, Mengarelli & Delichatsios, Michael A. \\
\hline Anysz, Hubert & Demidova, Liliya A. \\
\hline Asciuto, Antonio & Dhir, Amandeep \\
\hline Babkir, Ali & Díaz-Rodríguez, Miguel \\
\hline Bakhshinejad, Ali & Dong, Qinglin \\
\hline Bartlomiej, Oszczak & Dragisa, Stanujkic \\
\hline Basov, Suren & Duer, Stanisław \\
\hline Behrendt, Cezary & Dzedzickis, Andrius \\
\hline Bento-Gonçalves, António & Elahi, Ehsan \\
\hline Bocharova, Irina & Eliseev, Vladimir \\
\hline Bordel Sánchez, Borja & Escobedo, Pablo \\
\hline Borkovcová, Marie & Farhan, Laith \\
\hline BUI, Ngoc-Tam & Fikus, Bartosz \\
\hline Burlacu, Adrian & Frighi, Valentina \\
\hline Bylieva, Daria & Frigo, Oriel \\
\hline Cano-Ortega, Antonio & Gordon, Jeffrey \\
\hline Chand, Mohan Bahadur & Grigorescu, Adriana \\
\hline Chen, Shih-Chih & Hargreaves, Carol \\
\hline Chen, Yung Hsiang & Hölbl, Marko \\
\hline Cheraghian, Goshtasp & Holubek, Radovan \\
\hline Cherstvy, Andrey & Hong, Tao \\
\hline Cioccolanti, Luca & Hossain, Belayat \\
\hline Ciszkiewicz, Adam & Hu, Changjian \\
\hline Cmiljanic, Nikola & Hussain, Muhammad Imtiaz \\
\hline Cohen, Rami & Ikerionwu, Charles \\
\hline Costa, Carlos Miguel & Infante-Moro, Juan Carlos \\
\hline Costa, Susana & Irina, Mocanu \\
\hline
\end{tabular}


Islam, Saiful

Jamali, Seyed Hossein

Jamil, Faisal

János, Szenderák

Jiang, Guo

Jorge Luis Zambrano, Martinez

Karl, Christian Wolfgang

Karoń, Grzegorz

Karuppuswami, Saranraj

Kavadias, Kosmas

Khan, Irfan Ahmad

Khan, Md. Shakhaoath

Kouziokas, Georgios N.

Kováč, Viliam

Kracík, Petr

Krzywanski, Jaroslaw

Kumar, Arkadeep

Kuo, Wen-Ten

Lambán, María Pilar

Langmann, Reinhard

László, Vasa

Legendre, Tiffany

Lelea, Dorin

$\mathrm{Li}$, Chunguo

$\mathrm{Li}$, Tan

$\mathrm{Li}$, Tao

Li, Yuxing

Liang, Yi

Lin, Jeng-Chyan Muti

Linares-Barranco, Alejandro

Liu, Zhifu

Lu, Wei

Madsen, Dag Øivind

Mahian, Omid

Mandelli, Stefano

Mao, Lei

Mehrpouya, Mehrshad

Monteiro, João

Mosavi, IAmir

Nair, Gopika

Navid, Knejad

Nkenyereye, Lewis

Orehovački, Tihomir

Orkusz, Agnieszka

Otero, Pablo

P. Khope, Akhilesh S.

Pecchi, Matteo

Pellegrini, Marco

Peng, Jie

Pérez-García, Vicente

Peyman, Razi
Pienkowski, Krzysztof

Poór, Peter

Pouliakis, Abraham

Prieto, Jesús-Ignacio

Rafael Valotta, Rodrigues

Roy, Dhruvajyoti

Roy, Spandan

Ruz Ruiz, Mario Luis

Sabban, Albert

Saleem, Muhammad

Salehi, Amin

Saxena, Prateek

Schiavon, Michele

Schmid, Giovanni

Schmitt, Sebastian

Schneider, Lucas

Serna, Sergio

Sfica, Lucian

Shala, Ahmet

Shamshirband, Shahab

Sharma, Abhishek

Silaghi, Helga

Silvestri, Cecilia

Socas, Rafael

Song, Jifeng

Soto, Paul

Staszkiewicz, Piotr

Stauvermann, Peter Josef

Stevens, Gunnar

Tornabene, Francesco

Torrecilla Sánchez, Eva María

Tripathi, Takshashila

Tsuboi, Tsutomu

Tuclea, Claudia

Ullah, Md. Hafij

Ulloa Cerna, Alvaro

Verdini, Federica

Vojtovic, Sergej

Wang, Ning

Wang, Xianzhi

Wang, Zhuoying

Warmann, Emily

Watter, Holger

Witowski, Jan

Włodek, Tomasz

$\mathrm{Xu}$, Huijin

$X u$, Sheng

Xue, Bing

Yang, Huai

Yazdani Asrami, Mohammad

Zare, Mohsen 
Zhang, Lei

Zhang, Qi

Zhang, Qi

Zhang, Yu
Zhao, Xu-dong

Zhou, Yang

Zmywaczyk, Janusz

Zunino, Claudio 